EDUARD SPRANGER

Gesammelte Schriften

$\mathrm{X}$ 


\title{
Gesammelte Schriften
}

\author{
Herausgegeben von \\ Hans Walter Bähr Otto Friedrich Bollnow Otto Dürr \\ Walter Eisermann Ludwig Englert Andreas Flitner \\ Hermann Josef Meyer Hans Wenke †
}

$\mathrm{X}$

Max Niemeyer Verlag Tübingen
Quelle \& Meyer Verlag

Heidelberg 
EDUARD SPRANGER

\section{Hochschule und Gesellschaft}

Herausgegeben von

Walter Sachs

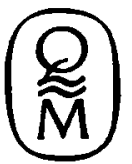

Quelle \& Meyer Verlag Heidelberg 1973 
Erscheint mit Unterstützung durch die Stiftung Volkswagenwerk, Hannover.

(C) 1973 Quelle \& Meyer - Heidelberg

Alle Rechte vorbehalten - Jede Vervielfältigung, gleich welcher Art und für welchen $Z$ wedk, ist ohne ausdrüdkliche Genehmigung des Verlags unzulässig. Satz und Drack: Drudkhaus Darmstadt GmbH. ISBN 3-494-00594-X 